\title{
Shape shifting
}

\author{
Faced with limited funding and the need to find the most time- and cost-efficient route to proof of concept and \\ relevance, how should you reorganize your company to facilitate development of your most valuable assets?
}

A miration mira was founded the same way as many other biotech companies today-by a group of talented scientists who wanted to pursue drug research their own way and on their own terms. The founders were seasoned drug hunters from Merck (Whitehouse Station, NJ, USA) who wanted to apply their knowledge of bioactive lipids, which had previously yielded such successful drugs as Singulair (montelukast), in the search for new drug candidates.

Like many other private biotech companies, Amira lived funding cycle to funding cycle, undertaking repeated rounds of fundraising to support R\&D efforts to obtain proof-ofconcept clinical data as rapidly and as cost effectively as possible. Faced with the lack of a vibrant initial public offering market and a post-mega-merger pharmaceutical world that limits partnership opportunities, let alone trade sale exits, Amira was forced to move into previously uncharted territory, trimming its broader research organization to focus on mid-stage clinical development. This transition was difficult, but looking back, I can say it was absolutely worth it.

\section{From partnership to acquisition}

In the early days of Amira, the focus was entirely on building a strong pipeline of investigational new drug (IND) application candidates. The team was successful at this endeavor and brought numerous therapeutic candidates into the clinic. But I knew that to be financially prudent Amira needed to focus only on the single best asset and build interest in it. I partnered our first program, a 5 -lipoxygenase-activating protein (FLAP) inhibitor, after completion of phase 1 studies in healthy volunteers, and planned to move into asthma and other respiratory diseases.

Bob Baltera, former CEO of Amira

Pharmaceuticals, San Diego, California, USA.

e-mail:info@panmira.com
Amira used the revenue generated by that partnership to develop other drug targets in rare diseases, which eventually attracted a buyer. Bristol-Myers Squibb (BMS) bought Amira for \$325 million upfront, and another \$150 million based on milestones around our phase 1 and preclinical fibrotic disease programs. This acquisition was a tremendous financial success, considering that Amira had raised only \$28 million in venture capital financing.

Partnering assets at early stages with major pharmaceutical companies is becoming more and more common, although success stories like Amira's are far outnumbered by failures. Amira's success can be credited to several factors. The Amira team had many years of experience in the pharmaceutical industry and understood the properties of a highquality drug candidate. Not only did the FLAP program have excellent pharmacokinetics and pharmacodynamics data, but it targeted asthma, a disease that has very few options for oral therapy. The tactic of partnering an asset early is especially relevant for companies developing products that target diseases served by the population of general practitioner physicians, also known as primary care, because development and commercialization of primary care products require enormous amounts of capital. Consequently, the new business model in the industry today is to partner with primary care programs as early as possible.

Amira was able to successfully follow this road map in its maturation as a company. Its FLAP inhibitor demonstrated potential in asthma and other large indications, so Amira was able to pursue and secure a major partnership with GlaxoSmithKline of London. This deal ensured that the FLAP program would have the necessary support to move forward, while also providing Amira with nondilutive financing for other inflammatory and fibrotic disease programs. Ultimately, the totality of all of these assets made Amira particularly attractive to BMS, and the Princeton,
New Jersey-based pharmaceutical company bought most of the company's assets in July 2011, giving our investors a lucrative exit of $\sim 15$ times the initial investment, if all milestones are reached. Other assets from the company, including a promising candidate for treatment of asthma and chronic obstructive pulmonary disease, were spun out into a new venture.

\section{Aligning visions}

There are many benefits for a company willing to move beyond R\&D and focus on mid-stage clinical development, the most important being a higher valuation for the programs-and the company itself_-as a clinical stage asset clears clinical hurdles. Although pharmaceutical companies have become more selective with partnerships and acquisitions in recent years, they have also been willing to pay higher premiums for programs with extensive validation, usually after clinical trials are completed. For example, moving a program from phase 1 into phase 2 studies will increase its value substantially for the sponsoring company.

In the case of Amira, this increased value benefited the founders and early investors of Amira, as it made additional follow-on efforts to raise capital and find a potential acquirer all the more likely. Indeed, it is critical for the management team of any young biotech company to understand the motivations and expectations of their investors, who are usually venture capitalists, before identifying a strategy and pathway for transition into a clinically capable organization. Companies that retain and develop assets can have substantial long-term growth and value creation. It may seem simple, but if investors and founders want to take a product to the market, then they first have to be willing to see it through early and mid-stage clinical development. It requires dedication, strong will and a sound financial plan to make this a reality. Partnering every program before clinical exploration does not support substantial growth over the life of the company. 


\section{Choosing the programs}

One of the first difficult steps is identifying which programs should be pursued, and which should be abandoned or put on the shelf. Companies founded on a strong platform technology or unique research capabilities may find themselves with a large number of promising IND candidates. Unfortunately, most companies have enough funding to take only one or two of those drugs into the clinic. Therefore, to make the transition to a clinically based company, a company needs to move forward with only the right programs. Determining which those are requires a robust analytical process that integrates scientific, medical, regulatory, marketing and financial information.

One of the first aspects to be evaluated is whether or not the drug candidate has the potential to be truly medically meaningful. Many times companies confuse good science with medically meaningful programs; however, they are different. The success of new therapies depends upon the ability to demonstrate meaningful outcomes for patients, providers and payors. Companies strive to understand these attributes before the expensive clinical trials begin. It is quite possible for incredibly innovative science to fall short on the medically meaningful scale. As such, advancing these types of programs may be a risky proposition. Many in our industry now use the term 'proof of relevance' to describe this requirement, and I believe it is here to stay (Box 1).

Although these steps may seem simple, they can often be missed by management teams focused only on the uniqueness of scientific programs and not their full potential for patients, providers and payors. Scientists are often very good at answering what makes interesting science, but many have limited experience when evaluating the clinical and economic benefit of a new therapy. It is important to have the right perspective (and diversity of thought) in the company when determining which programs are most deserving of transition into the clinic.

We faced these very decisions at Amira. It became clear that even though the INDgeneration engine had been successful, Amira could afford to pursue only some programs in the clinic. The new financial reality made this decision more challenging considering the fact that Amira was going to have to make this transition in a limited funding environment. Once Amira formed a successful partnership for our FLAP program, the management team decided to advance a novel drug candidate in fibrotic disease rather than pursue a clinical program for another asthma therapy.
This was not an easy decision. In the past, other biotech companies had moved fibrotic disease research into the clinic, but those programs proved to be unsuccessful. Fibrosis is generally viewed as a challenging disease to study in the clinic. I knew that our program needed to have strong medical and scientific support to have a better chance of moving forward. The program, a lysophosphatidic acid (LPA) receptor 1 antagonist, had a demonstrated link to fibrotic disease in clinical research, and had demonstrated potential in in vivo studies (Nat. Med. 14, 45-54, 2008). The good news about a challenging disease such as fibrosis is that it often does not engender a lot of competition. Importantly, Amira did not arrive at this decision to pursue fibrosis on its own. The company consulted numerous key opinion leaders, mostly in academia, all with extensive knowledge of fibrotic disease. These meetings were held as part of day-long events or by telephone with academic clinicians, physicians and researchers. All of this helped determine the pending clinical development plans.

\section{The staff}

Leaders of biotech companies must always understand the strengths and weaknesses of their team. It is important to assess any knowledge gaps and determine the best way possible to solve these issues. If a company is transitioning from a research-based knowledge center to a development-based knowledge core, restructuring may have to occur. This often involves reducing head count in research to add the needed knowledge base as well as preserve capital resources to invest in clinical development personnel and programs. These changes are often difficult to make, but they are necessary to ensure programs continue to move forward and to show that enterprise value is created.

Although this may sound like abandoning the original focus of the company, discovery operations can still continue, albeit in reduced form. Often, a discovery engine operating at full speed will reach a point where it generates more INDs than the company can afford to develop. Some of these programs may be potential candidates for development partnerships, though most will require at least some clinical development before a deal can be secured. The managerial juggling act is trying to find the right balance-keeping a smaller core research team in place to move early programs toward the clinic while still ensuring that there are the necessary funds to move clinical programs to a point where they can begin to generate value for shareholders. Successfully navigating this portfolio process and asset allocation is one of the most critical roles of a management team.

Amira faced this type of portfolio and organization structure decision in 2010 when it found itself with two programs in the clinic, a third ready to enter and several more moving forward. Without the funds to move all of these programs through mid-stage clinical development to secure partnerships, Amira had to change focus. We reduced head count in

\section{Box 1 Proof of relevance}

Proof of concept is widely recognized as one of the most important milestones in drug development. In fact, reaching this point can become the singular focus of a team that is looking to develop therapeutics - either for partnerships or commercialization on its own. Proof of concept is the moment in time in which a company can look at clinical data and state with confidence that their experimental therapy is demonstrating an impact on the underlying biology that it is targeting. Although this is an important milestone to achieve, especially when seeking partnerships, companies looking to make an impact with a therapeutic candidate cannot overlook the importance of also demonstrating proof of relevance.

Although proof of concept applies wholly to the science itself, proof of relevance is an evaluation of how useful a potential therapeutic will be in a specific disease. This includes identifying medical need and understanding how the therapeutic candidate will potentially address it. The therapeutic candidate must have the potential to address this unmet need in an efficient enough manner to be used by physicians and reimbursed by payers. These hurdles are critically important to persuade major drug developers to pay significant fees to in-license such candidates or for investors to fund the development.

The growing demand for proof of relevance does not replace the demand for proof of concept, as both are often necessary to secure partnerships or achieve commercial success. Developing therapeutics that clear both bars is the best path to having a successful therapeutic, either through internal development or through a partnership, and making an impact for healthcare and patients. 
discovery to allow investment in clinical development. The core research team retained in the company was focused on advancing existing programs and was not pursuing new drug targets. The decision was difficult, but necessary to ensure success for the organization. At the same time, however, it is important to stress that retaining the core scientific talent focused on LPA1 and other fibrosis targets made Amira an ideal target for an acquisition. BMS has stated that they intend to retain the Amira scientific staff to continue work in this area.

\section{Conclusions}

The decisions that face emerging biotech companies today are challenging, yet necessary. Although it can be difficult to limit the number of programs moving forward and to reduce head count, these steps can be necessary to ensure the company has a viable future and is an ideal candidate for a partnership or acquisition. Our industry is about transforming scientific ideas and discoveries into meaningful therapeutic options. It is important to keep in mind that our efforts are for the benefit of patients and their families. The financial reality and processes involved in building successful biotech companies may have changed, but the end result remains worth the difficult decisions made.

For more content on bioentrepreneurism, visit our Trade Secrets blog

http://blogs.nature.com/trade_secrets/ 